\title{
Peroxisome proliferator-activated receptor- $\gamma$ inhibits pancreatic cancer cell invasion and metastasis via regulating MMP-2 expression through PTEN
}

\author{
YUE LI, DA-WEI ZHANG, DIAN-QIANG LIN and LIANG-QI CAO \\ Department of Hepatobiliary Surgery, The Second Affiliated Hospital of Guangzhou Medical University, \\ Guangzhou, Guangdong 510260, P.R. China
}

Received October 20, 2014; Accepted July 17, 2015

DOI: $10.3892 / \mathrm{mmr} .2015 .4224$

\begin{abstract}
The invasive and metastatic behavior of pancreatic cancer is associated with a poor prognosis. Therefore, understanding the molecular mechanisms underlying the invasion and metastasis of pancreatic cancer has important application values theoretically and clinically. In previous years, with increasing studies focusing on tumor pathogenesis, it has been revealed that peroxisome proliferator-activated receptor- $\gamma$ $(\mathrm{PPAR} \gamma)$ and phosphatase and tensin homolog (PTEN) are closely associated with the occurrence and development of pancreatic cancer. Thus, in the present study, a scratch wound assay, western blotting and transwell assays were used to investigate their function. The scratch wound assay demonstrated that treatment with the PPAR $\gamma$ ligand rosiglitazone (RGZ) could reduce the movement and migration of pancreatic cancer cells. Western blotting results indicated that while RGZ inhibited the expression of matrix metalloproteinase (MMP)-2, PPAR $\gamma$ inhibitors promoted MMP-2 expression. However, PPAR $\gamma$ ligands and inhibitors did not affect the expression of MMP-9. Further investigation indicated that the regulation of MMP-2 by PPAR $\gamma$ activation occurred through PTEN. In addition, PPAR $\gamma$ activation promoted PTEN expression, thereby inhibiting the expression of MMP-2. Subsequent transwell experiments demonstrated that RGZ treatment significantly inhibited the invasiveness of pancreatic cancer cells and the inhibitory effect of RGZ was completely reversed by simultaneous transfection of the MMP-2-overexpressing vector, which increased the invasiveness of pancreatic cancer cells. Therefore, PPAR $\gamma$ activation can activate PTEN
\end{abstract}

Correspondence to: Dr Liang-Qi Cao, Department of Hepatobiliary Surgery, The Second Affiliated Hospital of Guangzhou Medical University, 250 Changgang East Road, Haizhu, Guangzhou, Guangdong 510260, P.R. China

E-mail: clq0829@163.com

Key words: peroxisome proliferator-activated receptor- $\gamma$, pancreatic cancer cells, matrix metalloproteinase-2, phosphatase and tensin homolog, rosiglitazone expression, thereby suppressing the expression of MMP-2 and hence inhibiting the invasion and metastasis of pancreatic cancer cells.

\section{Introduction}

Pancreatic carcinoma is a malignant tumor of the digestive system and has an extremely poor prognosis. The average survival time following diagnosis is $<6$ months and the 5-year survival rate is $<5 \%$ (1). In China, the incidence of pancreatic cancer is increasing every year and only a small number of patients are able to undergo surgical resection. However, there remains a high recurrence rate and a poor prognosis $(1,2)$. Invasion and metastasis are the key factors impacting the recurrence and mortality of patients with pancreatic carcinoma. Therefore, identifying effective molecular targets to prevent or reduce pancreatic cancer invasion and metastasis is critical for improving the treatment efficacy and prognosis.

Peroxisome proliferator-activated receptor- $\gamma$ (PPAR $\gamma)$ belongs to a class of ligand-activated nuclear transcription factors and is a member of the type II nuclear receptor superfamily (3). PPAR $\gamma$ ligands are divided into two categories as natural ligands, including 15-deoxy prostaglandin J2 (15d-PGJ2), and synthetic ligands, including mainly thiazolidinedione compounds, such as rosiglitazone (RGZ), pioglitazone (PGZ) and troglitazone (TGZ). Among the synthetic ligands, RGZ has the highest bioavailability and efficacy and the least adverse effects. Previous in vivo experiments demonstrated that following activation with ligands, PPAR $\gamma$ can inhibit tumor cell growth, induce apoptosis of tumor cells and exert other biological effects $(3,4)$. Sasaki et al (5) detected the expression of PPAR $\gamma$ in human pancreatic cancer cells and human pancreatic tissues using western blot analysis and reverse transcription polymerase chain reaction (RT-PCR) methods. The authors found that PPAR $\gamma$ was expressed in all human pancreatic cancer cells and $71 \%$ of human pancreatic cancer tissues, while no PPAR $\gamma$ expression was observed in the adjacent normal pancreatic tissues. Farrow et al (6) used RGZ to activate PPAR $\gamma$ in the pancreatic cancer cell line AsPC-1 and thereby suppressed the invasiveness of AsPC-1 cells via reducing the secretion of a tumor cell invasion factor i.e. tissue plasminogen activator. Thus, PPAR $\gamma$ activation can inhibit the 
invasion of pancreatic cancer, though the exact mechanism remains to be elucidated.

Hashimoto et al (7) found that the PPAR $\gamma$ natural ligand 15d-PGJ2 can suppress the expression of MMP-2 and MMP-9 and inhibit pancreatic cancer cell invasion. MMPs are a group of calcium- and zinc-dependent proteases, and together with their inhibitor, tissue inhibitor of metalloproteinases (TIMPs), regulate tumor invasion and metastasis. Among them, MMP-2 and MMP-9 were found to be overexpressed in pancreatic cancer tissue and closely associated with tumor invasion and metastasis $(8,9)$. In addition, Farrow et al $(10)$ reported that the selective PPAR $\gamma$ agonist RGZ enhanced the expression of phosphatase and tensin homolog (PTEN) in pancreatic cancer AsPC-1 cells and inhibited AKT phosphorylation, while application of the PPAR $\gamma$ inhibitor GW9662 suppressed PTEN expression, suggesting that PPAR $\gamma$ ligands attenuate pancreatic cancer cell growth via inhibiting phosphoinositide 3-kinase (PI3K) activity through upregulating the expression of the tumor suppressor gene PTEN. PTEN was identified in 1997 as a tumor suppressor gene closely associated with cancer (11) w. It is important in tumor cell apoptosis and migration and is the main component of signaling pathways that control cell cycle initiation and exit, promote differentiation, initiate repair and regulate apoptosis (11). Therefore, PPAR $\gamma$ activation may possibly regulate the expression of MMPs through PTEN and thereby inhibit the invasion of pancreatic cancer cells. These aspects were examined in the present study.

\section{Materials and methods}

Cell culture. Human pancreatic carcinoma PANC-1 cell lines were purchased from the American Type Culture Collection and were maintained in Dulbecco's modified Eagle's medium (Gibco-BRL, Carlsbad, CA, USA) supplemented with 10\% fetal bovine serum (Gibco-BRL), $100 \mathrm{U} / \mathrm{ml}$ penicillin and $100 \mu \mathrm{g} / \mathrm{ml}$ streptomycin. Cells were maintained at $37^{\circ} \mathrm{C}$ in a humidified atmosphere containing $5 \% \mathrm{CO}_{2}$.

Overexpressionvectorand smallinterfering RNA(siRNA)transfection. The following primers were used for PTEN and MMP-2 CDS amplification: PTEN-CDS, forward 5'-CGGAATTCG CCACCATGACAGCCATCATCAAAGAG-3' and reverse 5'-CCGCTCGAGTCAGACTTTTGTAATTTGTGTATGC-3'; MMP2-CDS, forward 5'-CGGAATTCGCCACCATGGAGG CGCTAATGGCCCG-3' and reverse 5'-CCGCTCGAGTCA GCAGCCTAGCCAGTCGG-3'. Underlined letters represent the restriction enzyme sites, and bold letters represent the Kozak sequence. The products were digested with EcoRI and XhoI (Takara Bio, Inc., Otsu, Japan), cloned into pcDNA3.1 vectors, sequenced and verified. PTEN and negative control (NC) siRNAs were obtained from Santa Cruz Biotechnology, Inc. (Shanghai, China). The cells were seeded into 6-well plates at $1 \times 10^{5}$ cells $/ \mathrm{ml}$ and incubated for $24 \mathrm{~h}$. When the cell confluence reached $\sim 70 \%$, plasmid and siRNA transfection were performed according to the manufacturer's instructions of Lipofectamine 2000 (Invitrogen Life Technologies, Carlsbad, CA, USA). The medium was changed within 4-6 $\mathrm{h}$ after transfection. After $24 \mathrm{~h}$ of transfection, either RGZ $(20 \mu \mathrm{M})$ or GW9662 $(20 \mu \mathrm{M})$ was added and the cells were cultured for $24-48 \mathrm{~h}$.
Western blot analysis. Total cellular proteins were extracted by incubating cells in lysis buffer. The protein concentrations in the cell lysates were determined by bicinchoninic acid assay (Pierce Biotechnology Inc., Rockford, IL, USA). SDS-PAGE was performed in $8 \%$ glycine gels (Bio-Rad Laboratories Inc., Hercules, CA, USA) loading equal quantities of proteins per lane. Following electrophoresis, separated proteins were transferred onto nitrocellulose membranes and blocked with 5\% non-fat milk in Tris-buffered saline and Tween 20 for $1 \mathrm{~h}$. Following this, the membranes were incubated with rabbit anti-human polyclonal MMP-2 (cat. no. 4022; Cell Signaling Technology, Inc., Beverly, MA, USA; 1:1,000), rabbit anti-human polyclonal MMP-9 (cat. no. 3852; Cell Signaling Technology, Inc.; 1:1,000), rabbit anti-human monoclonal PTEN (cat. no. 9552; Cell Signaling Technology Inc.; 1:600) and mouse anti-human monoclonal GAPDH (cat. no. NBP1-47339; Novus Biologicals, San Diego, CA, USA; 1:1,000) overnight at $4^{\circ} \mathrm{C}$, and then secondary antibodies conjugated with horseradish peroxidase (anti-rabbit IgG; cat. no. 7074; Cell Signaling Technology, Inc.) at 1:7,000-8,000 dilution were incubated with membranes for $1 \mathrm{~h}$ at room temperature. Protein bands were detected using the West Femto System (Pierce Biotechnology, Inc.).

Wound healing assay. Cells $\left(1 \times 10^{6}\right.$ per well) were seeded in 6 -well plates and allowed to grow until 70\% confluence. The cells were pretreated with Mitomycin C (Roche Diagnostics, Hong Kong, China), which inhibits cell division, so that the difference in motility was not affected by differences in cell proliferation rates. The cells were treated as above and monolayers were then wounded with a pipette tip. Following being washed with phosphate buffered-saline (PBS) three times, the Olympus IX-70 microscope (Olympus, Tokyo, Japan) was used to capture images immediately following scratching for $0,6,24$ and $48 \mathrm{~h}$ in the same marked location of the dish. Data were obtained from three independent experiments.

Transwell matrigel invasion assay. Invasion of cells was evaluated by Transwell matrigel invasion assay. Briefly, $200 \mu \mathrm{l}$ of cells following transfection $\left(1 \times 10^{6}\right.$ cells $\left./ \mathrm{ml}\right)$ and $600 \mu 1$ of the complete medium were added to the upper and lower compartments of the chamber, respectively. Following incubation for $48 \mathrm{~h}$, cells migrating to the lower side of the filter were fixed with $4 \%$ paraformaldehyde for $15 \mathrm{~min}$ at room temperature, washed with PBS, stained with crystal violet and then observed under an Olympus Fluoview 500 IX71 confocal microscope (Olympus).

Statistical analysis. The experiments were performed at least in triplicate and results are expressed as the mean \pm standard deviation. SPSS statistical package (SPSS 13.0 for Windows; SPSS, Inc., Chicago, IL, USA) was used for statistical analysis. The difference between two groups was analyzed by two-tailed Student's t-test and that between three or more groups was analyzed by one-way analysis of variance multiple comparisons. $\mathrm{P}<0.05$ and $\mathrm{P}<0.01$ were considered to indicate a statistically significant difference.

\section{Results}

PPAR $\gamma$ activation by RGZ inhibits the migration of pancreatic cancer cells. The majority of current studies suggest that 

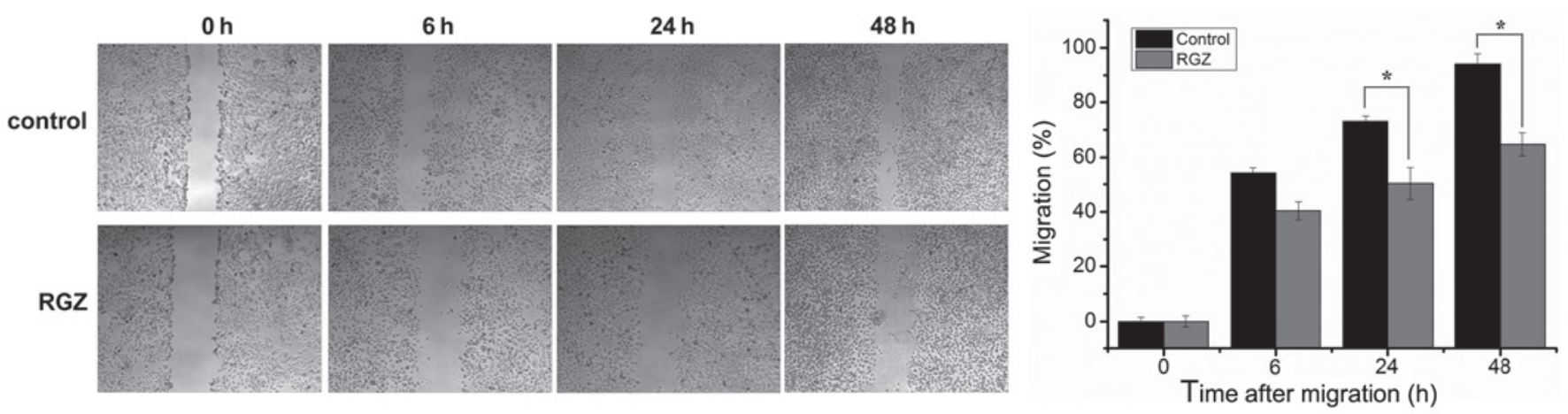

Figure 1. RGZ reduces pancreatic cancer cell migration in vitro. Each bar represents the mean \pm standard deviation from three samples ("P<0.01). RGZ, rosiglitazone.
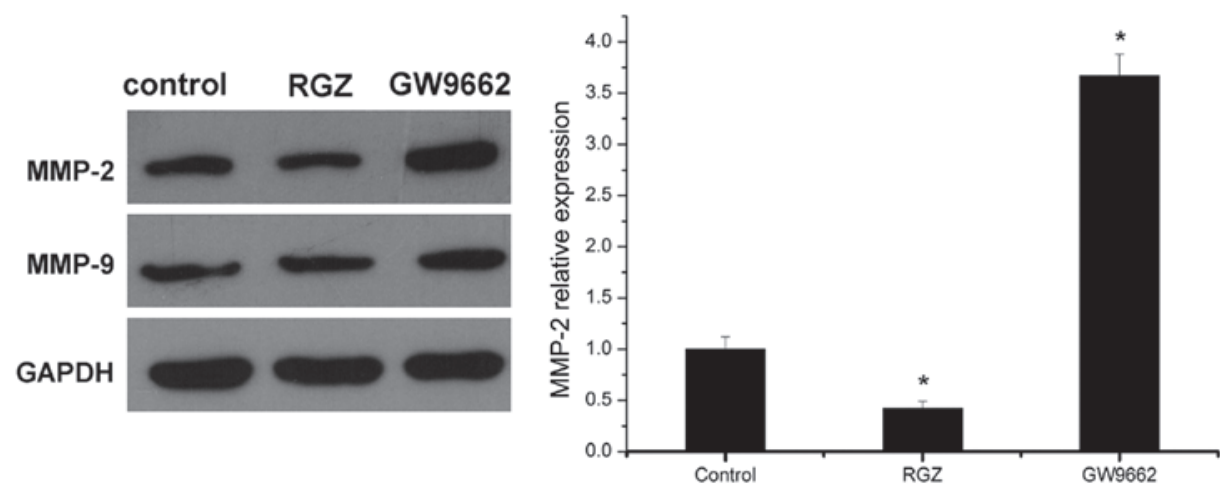

Figure 2. Expression of MMP-2 and MMP-9 in PANC-1 cells. RGZ (20 $\mu \mathrm{M})$ or GW9662 (20 $\mu \mathrm{M})$ was added to PANC-1 cells and cultured for $48 \mathrm{~h}$. Subsequently, the expression of MMP-2 and MMP-9 was measured by western blot analysis. The results are presented as the mean \pm standard deviation ( $\mathrm{n}=3$ ). "P<0.01 vs. control. RGZ, rosiglitazone; MMP, matrix metalloproteinase.

PPAR $\gamma$ activation can inhibit the migration and invasion of tumor cells, however, few relevant studies have focused on pancreatic cancer. The present study initially demonstrated the effect of RGZ on the in vitro migration ability of the pancreatic cancer cell line Panc-1 using a scratch wound assay. The cells were subjected to scratch tests following RGZ treatment and the scratch wound was monitored following different periods of time. The results shown in Fig. 1 indicated that the migration of RGZ-treated cells was markedly slower than that of the control cells at the indicated time points. These results suggested that in pancreatic cancer cells, RGZ activation of PPAR $\gamma$ has the ability to inhibit migration.

PPAR $\gamma$ activation regulates MMP-2 expression in pancreatic cancer cells. During the process of invasion and metastasis, tumor cells must overcome a series of natural barriers, including the extracellular matrix (ECM). MMPs are the main direct factors involved in the destruction of the ECM; among them, type II collagenases, including MMP-2 and MMP-9, are most closely associated with tumor invasion and metastasis (12). Therefore, the present study further examined the association between PPAR $\gamma$ activation and the expression of MMP-2 and MMP-9. The results demonstrated that RGZ treatment significantly inhibited MMP-2 expression, whereas addition of the PPAR $\gamma$-specific inhibitor GW9662 significantly upregulated the expression of MMP-2. However, neither RGZ nor GW9662 significantly altered the expression of MMP-9 (Fig. 2), suggesting that in pancreatic cancer cells, PPAR $\gamma$ activation predominantly affected the expression of MMP-2, while MMP-9 expression may be regulated by other factors.

PPAR $\gamma$ activation regulates MMP-2 expression through PTEN. Existing studies have demonstrated that PPAR $\gamma$ activation exerts an inhibitory effect on tumor growth by activating the tumor suppressor gene PTEN $(10,11)$. In addition, several studies have also indicated that PTEN has a tumor suppressor role via regulating a series of factors, including MMP-2 $(11,13,14)$. Therefore, PPAR $\gamma$, PTEN and MMP-2 are closely associated with each other. Following transfection of a PTEN-overexpressing vector into cells, the expression of MMP-2 was downregulated. When treating the pancreatic cancer cells with RGZ, upregulation of PTEN expression occurred and the expression of MMP-2 was simultaneously downregulated. However, following transfection of PTEN siRNA, the RGZ-induced upregulation of PTEN expression was inhibited and the inhibitory effect of RGZ on MMP-2 expression was also reversed (Fig. 3). These results indicated that PPAR $\gamma$ activation inhibits MMP-2 expression via activating PTEN.

PPAR $\gamma$ activation affects pancreatic cancer cell invasion by regulating the expression of $M M P-2$. PPAR $\gamma$ activation affects the invasion and metastasis of pancreatic cancer cells, 


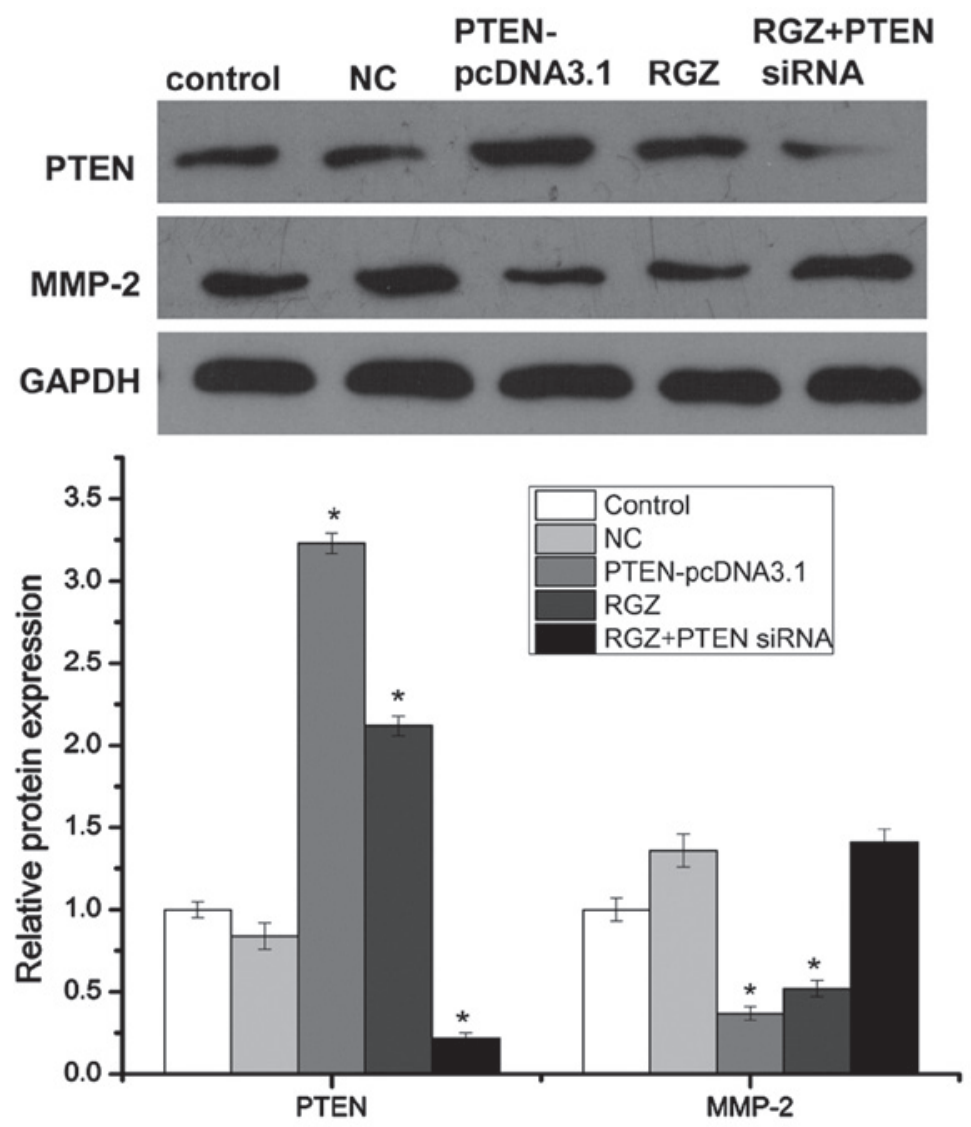

Figure 3. Expression of PTEN and MMP-2 in PANC-1 cells. PANC-1 cells were either transfected with a PTEN-overexpressing vector, treated with RGZ $(20 \mu \mathrm{M})$ or transfected with PTEN siRNA and treated with RGZ $(20 \mu \mathrm{M})$. The expression of PTEN and MMP-2 was then measured by western blot analysis. The results are presented as the mean \pm standard deviation $(n=3)$. ${ }^{*} \mathrm{P}<0.01$ vs. control. RGZ, rosiglitazone; MMP, matrix metalloproteinase; PTEN, phosphatase and tensin homolog; siRNA, small interfering RNA; NC, negative control.
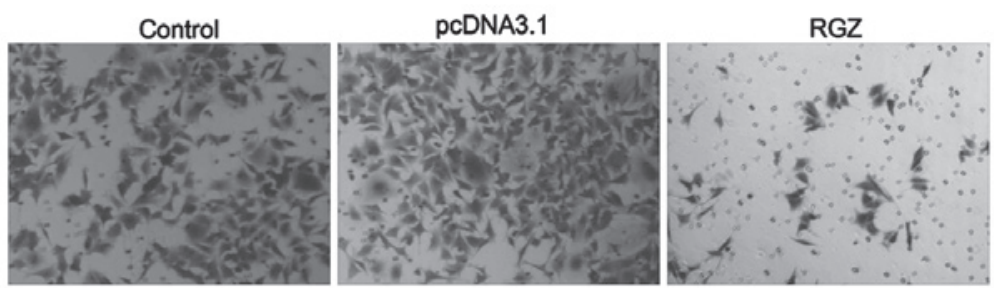

RGZ+MMP-2-pcDNA3.1

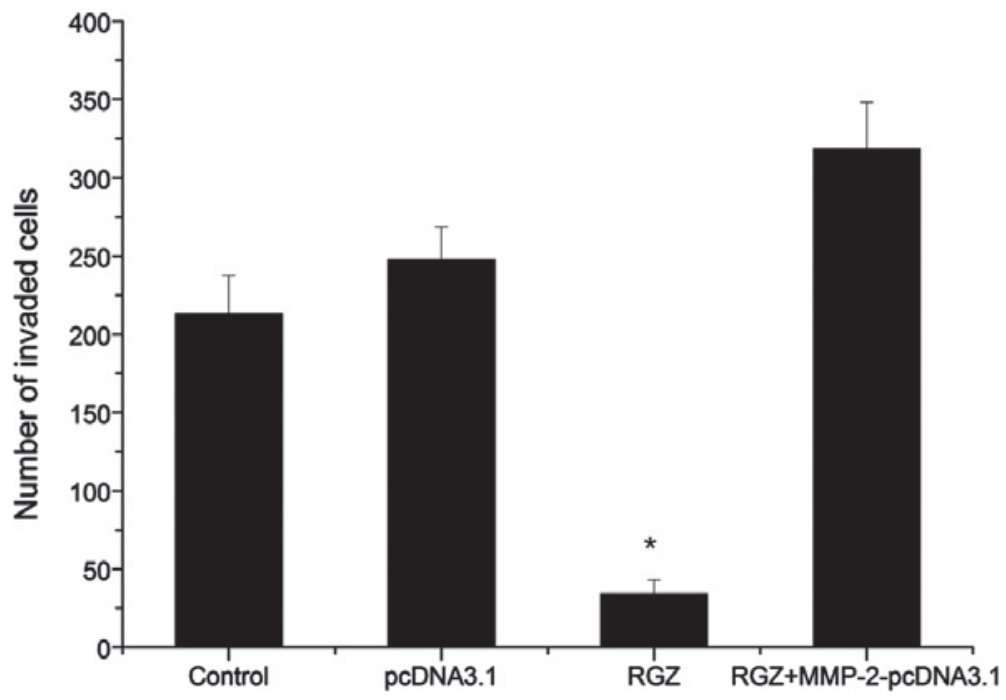

Figure 4. Effect of RGZ and MMP-2 on PANC-1 cell invasion. Transwell analysis of PANC-1 cells following different treatments. The relative ratios of invasive cells per field is shown. Each bar represents the mean \pm standard deviation from three samples ("P $<0.01$ vs. control). RGZ, rosiglitazone; MMP, matrix metalloproteinase. 
and MMP-2 is closely associated with tumor cell invasion and metastasis. Therefore, in order to determine whether PPAR $\gamma$ activation affects the invasion of pancreatic cancer cells via regulating MMP-2 expression, further investigation was conducted. The results of the transwell experiment demonstrated that RGZ significantly inhibited the invasiveness of pancreatic cancer cells. However, transfection with a vector overexpressing MMP-2 completely suppressed the inhibitory effect of RGZ on pancreatic cancer cell invasion, and the invasiveness of pancreatic cancer cells exhibited an increasing trend (Fig. 4), indicating that PPAR $\gamma$ activation can affect the invasion and metastasis of pancreatic cancer cells via regulating MMP-2 expression.

\section{Discussion}

In previous years, the incidence of pancreatic cancer has exhibited a rising trend globally. It has a latent onset and rapid progression, but there is are few effective methods for an early diagnosis and systemic treatment. Due to the late diagnosis and poor treatment level of pancreatic cancer there is an urgent requirement for new and effective molecular targets for the treatment of pancreatic cancer (15).

PPARs belong to the nuclear receptor gene superfamily, containing three subtypes: $\alpha, \beta$ (or $\delta$ ) and $\gamma$. Among them, PPAR $\gamma$ is the most frequently investigated and is closely associated with tumors. Previous studies have demonstrated that upon activation with specific ligands, PPAR $\gamma$ can inhibit the growth of human tumor cells in vitro and in vivo $(10,11,16)$. PPAR $\gamma$ and its ligands have thus become one of the main focuses of basic tumor research. Currently, the results of studies on PPAR $\gamma$ in pancreatic cancer have demonstrated that PPAR $\gamma$ is highly expressed in pancreatic tumor tissues (17) and PPAR $\gamma$ activation by ligands inhibits the growth and arrests the cell cycle of human pancreatic cancer cells at the G1 phase through various pathways (5,18-20). Itami et al (20) found that overexpression of PPAR $\gamma$ in the pancreatic cancer cell line KMP-3 led to lipid accumulation, while treatment with TGZ increased the level of p27Kip1 and thereby arrested the cell cycle at G1 phase. However, few studies have focused on the association between PPAR $\gamma$ and pancreatic cancer cell invasion and metastasis. A scratch wound assay in the present study demonstrated that following treatment with RGZ, pancreatic cancer cells exhibited decreased movement and migration, which is consistent with current findings in other cell lines. For example, the in vitro and in vivo experiments of $\mathrm{Yu}$ et al (21) identified that RGZ inhibited the invasion and metastasis of human hepatocellular carcinoma cells MHCC97-L and BEL-7404. Therefore, similarly, RGZ activation of PPAR $\gamma$ can inhibit the migration of pancreatic cancer cells.

The invasion and metastasis of pancreatic cancer cells is a continuous multi-step process, an important step of which is the detachment of tumor cells from the primary site by degrading the basal membrane (BM) and ECM through secretion of destructive enzymes; the tumor cells then migrate through the ECM, invade the circulation, and form metastatic lesions. The MMPs and TIMPs together regulate the integrity of the BM and ECM $(12,22)$. Several studies have demonstrated that compared with normal pancreatic tissue, subcutaneously-implanted hamster pancreatic ductal carcinoma cells overexpress MMP-2 and MMP-9, and the serum levels of MMP-2 and MMP-9 also increased significantly. The growth of pancreatic cancer was significantly correlated with serum MMP levels (23). Another study demonstrated that among 32 pancreatic resection specimens, 31 were found with venous invasion. Compared with patients without hepatic metastasis, cases with hepatic metastasis demonstrated a significantly increased invasion of large and medium vessels, which was correlated with the overexpression of MMP-2 and MMP-9 (24). Therefore, MMP-2 and MMP-9 has an important role in the invasion and metastatic process of pancreatic cancer. The present study found that RGZ significantly downregulated the expression of MMP-2 in pancreatic cancer cells, whereas the PPAR $\gamma$ specific inhibitor GW9662 was able to promote MMP-2 expression. However, the effect of RGZ and GW9662 on MMP-9 expression was not significant. These results suggested that in pancreatic cancer cells, PPAR $\gamma$ activation predominantly affects the expression of MMP-2, thereby affecting invasion and metastasis, which was further confirmed by a subsequent transwell test. The results of the transwell experiment demonstrated that RGZ significantly inhibited the invasiveness of pancreatic cancer cells. However, transfection with a vector overexpressing MMP-2 completely suppressed the inhibitory effect of RGZ on pancreatic cancer cell invasion, and the invasiveness of pancreatic cancer cells exhibited an increasing trend, indicating that PPAR $\gamma$ activation can affect the invasion and metastasis of pancreatic cancer cells via regulating MMP-2 expression. Using immunoblotting and RT-PCR methods, Sasaki et al (5) demonstrated that the PPAR $\gamma$ ligand 15d-PGJ2 significantly inhibited the infiltration of pancreatic cancer cells, which was associated with a reduced activity and expression of MMP-2 and MMP-9.

In addition, a previous study has also demonstrated that PPAR $\gamma$ activation increases the expression of PTEN, the first tumor suppressor gene identified with phosphatase activity, and can antagonize the activation of protein kinase $B$ by PI3K, inhibit the Akt/PI3K signaling pathway, regulate cell proliferation and the cell cycle and inhibit cell growth, thus having a role in tumor cell metastasis (25). In previous years, with increasing studies on tumor pathogenesis, it has been revealed that PPAR $\gamma$ and PTEN are closely associated with the occurrence and development of pancreatic cancer $(20,21)$. Therefore, whether PPAR $\gamma$ activation regulates the expression of MMP-2 via PTEN was investigated and the data confirmed that PPAR $\gamma$ activation inhibited the expression of MMP-2 through activating PTEN. Galli et al (26) found that thiazolidinediones inhibited the invasiveness of pancreatic cancer cells, affecting gelatinolytic and fibrinolytic activity with a mechanism involving MMP-2 and PAI-1 expression.

In conclusion, RGZ activates PPAR $\gamma$ leading to increased PTEN expression and consequently inhibits the expression of MMP-2, thereby inhibiting the invasion and metastasis of pancreatic cancer cells. The results further illustrated the mechanism underlying the inhibitory effect of PPAR $\gamma$ activation on the invasion and metastasis of pancreatic cancer cells, and hence provided sufficient scientific basis to establish the PPAR $\gamma$ signaling pathway as a new target for pancreatic cancer therapy. 


\section{Acknowledgements}

This study was supported by research grants from the Science and Technology Project in Guangdong Province (grant no. 2012B061700047) and the Science and Technology Project in Guangzhou City-Zhujiang Science and Technology New Star (grant no. 2012J2200039). The authors would like to thank Guang Zhou Ying Sen Biotech Co., Ltd.

\section{References}

1. Yachida $\mathrm{S}$ and Iacobuzio-Donahue CA: The pathology and genetics of metastatic pancreatic cancer. Arch Pathol Lab Med 133: 413-422, 2009.

2. Huguet F, Girard N, Guerche CS, Hennequin C, Mornex F and Azria D: Chemoradiotherapy in the management of locally advanced pancreatic carcinoma: A qualitative systematic review. J Clin Oncol 27: 2269-2277, 2009.

3. Koeffler HP: Peroxisome proliferator-activated receptor gamma and cancers. Clin Cancer Res 9: 1-9, 2003.

4. Rumi MA, Sato H, Ishihara S, Kawashima K, Hamamoto S, Kazumori H, Okuyama T, Fukuda R, Nagasue $\mathrm{N}$ and Kinoshita Y: Peroxisome proliferator-activated receptor gamma ligand-induced growth inhibition of human hepatocellular carcinoma. Br J Cancer 84: 1640-1647, 2001

5. Sasaki T, Fujimoto Y, Tsuchida A, Kawasaki Y, Kuwada Y and Chayama K: Activation of peroxisome proliferator-activated receptor gamma inhibits the growth of human pancreatic cancer. Pathobiology 69: 258-265, 2001.

6. Farrow B, O'Connor KL, Hashimoto K, Iwamura T and Evers BM: Selective activation of PPARgamma inhibits pancreatic cancer invasion and decreases expression of tissue plasminogen activator. Surgery 134: 206-212, 2003.

7. Hashimoto K, Ethridge RT and Evers BM: Peroxisome proliferator-activated receptor gamma ligand inhibits cell growth and invasion of human pancreatic cancer cells. Int J Gastrointest Cancer 32: 7-22, 2002.

8. Koshiba T, Hosotani R, Wada M, Fujimoto K, Lee JU, Doi R, Arii $\mathrm{S}$ and Imamura M: Detection of matrix metalloproteinase activity in human pancreatic cancer. Surg Today 27: 302-304, 1997.

9. Bramhall SR, Neoptolemos JP, Stamp GW and Lemoine NR: Imbalance of expression of matrix metalloproteinases (MMPs) and tissue inhibitors of the matrix metalloproteinases (TIMPs) in human pancreatic carcinoma. J Pathol 182: 347-355, 1997.

10. Farrow B and Evers BM: Activation of PPARgamma increases PTEN expression in pancreatic cancer cells. Biochem Biophys Res Commun 301: 50-53, 2003.

11. Chen JS, Wang Q, Fu XH, Huang XH, Chen XL, Cao LQ, Chen LZ, Tan HX, Li W, Bi J, et al: Involvement of $\mathrm{PI} 3 \mathrm{~K} / \mathrm{PTEN} / \mathrm{AKT} / \mathrm{mTOR}$ pathway in invasion and metastasis in hepatocellular carcinoma: Association with MMP-9. Hepatol Res 39: 177-186, 2009.

12. Nagase H and Woessner JF Jr: Matrix metalloproteinases. J Biol Chem 274: 21491-21494, 1999.
13. Li X, Yang Z, Song W, Zhou L, Li Q, Tao K, Zhou J, Wang X, Zheng Z, You N, Dou K and Li H: Overexpression of Bmi-1 contributes to the invasion and metastasis of hepatocellular carcinoma by increasing the expression of matrix metalloproteinase (MMP)-2, MMP-9 and vascular endothelial growth factor via the PTEN/PI3K/Akt pathway. Int J Oncol 43:793-802, 2013.

14. Tian T, Nan KJ, Guo H, Wang WJ, Ruan ZP, Wang SH, Liang X and Lu CX: PTEN inhibits the migration and invasion of HepG2 cells by coordinately decreasing MMP expression via the PI3K/Akt pathway. Oncol Rep 23: 1593-600, 2010.

15. Goggins M: Molecular markers of early pancreatic cancer. J Clin Oncol 23: 4524-4531, 2005.

16. Michalik L, Desvergne $B$ and Wahli W: Peroxisome-proliferator-activated receptors and cancers: Complex stories. Nat Rev Cancer 4: 61-70, 2004.

17. Kristiansen G, Jacob J, Buckendahl AC, Grützmann R, Alldinger I, Sipos B, Klöppel G, Bahra M, Langrehr JM, Neuhaus P, et al: Peroxisome proliferator-activated receptor gamma is highly expressed in pancreatic cancer and is associated with shorter overall survival times. Clin Cancer Res 12: 6444-6451, 2006.

18. Toyota M, Miyazaki Y, Kitamura S, Nagasawa Y, Kiyohara T, Shinomura Y and Matsuzawa Y: Peroxisome proliferator-activated receptor gamma reduces the growth rate of pancreatic cancer cells through the reduction of cyclin D1. Life Sci 70: 1565-1575, 2002.

19. Kawa S, Nikaido T, Unno H, Usuda N, Nakayama K and Kiyosawa K: Growth inhibition and differentiation of pancreatic cancer cell lines by PPAR gamma ligand troglitazone. Pancreas 24: 1-7, 2002.

20. Itami A, Watanabe G, Shimada Y, Hashimoto Y, Kawamura J, Kato M, Hosotani R and Imamura M: Ligands for peroxisome proliferator-activated receptor gamma inhibit growth of pancreatic cancers both in vitro and in vivo. Int $\mathrm{J}$ Cancer 94: 370-376, 2001

21. Yu J, Shen B, Chu ES, Teoh N, Cheung KF, Wu CW, Wang S, Lam CN, Feng $\mathrm{H}$, Zhao J, et al: Inhibitory role of peroxisome proliferator-activated receptor gamma in hepatocarcinogenesis in mice and in vitro. Hepatology 51: 2008-2019, 2010.

22. Gomez DE, Alonso DF, Yoshiji H and Thorgeirsson UP: Tissue inhibitors of metalloproteinases: Structure, regulation and biological functions. Eur J Cell Biol 74: 111-122, 1997.

23. Iki K, Takeo T, Kubozoe T, Aoki S, Hayashi J and Tsunoda T: Detection of serum MMPs in tumor-bearing hamsters. J Hepatobiliary Pancreat Surg 9: 478-484, 2002.

24. Nagakawa Y, Aoki T, Kasuya K, Tsuchida A and Koyanagi Y: Histologic features of venous invasion, expression of vascular endothelial growth factor and matrix metalloproteinase-2 and matrix metalloproteinase- 9 and the relation with liver metastasis in pancreatic cancer. Pancreas 24: 169-178, 2002.

25. Jones G, Machado J Jr, Tolnay M and Merlo A: PTEN-independent induction of caspase-mediated cell death and reduced invasion by the focal adhesion targeting domain (FAT) in human astrocytic brain tumors which highly express focal adhesion kinase (FAK). Cancer Res 61: 5688-5691, 2001.

26. Galli A, Ceni E, Crabb DW, Mello T, Salzano R, Grappone C, Milani S, Surrenti E, Surrenti C and Casini A: Antidiabetic thiazolidinediones inhibit invasiveness of pancreatic cancer cells via PPARgamma independent mechanisms. Gut 53: 1688-1697, 2004. 